\title{
Development and Pilot Evaluation of the ArmTracker: A Wearable System to Monitor Arm Kinematics During Daily Life
}

\author{
Víctor A. Carmona-Ortiz ${ }^{\dagger}$, Joan Lobo-Prat ${ }^{\dagger}$, Jeremy Van Ruysevelt \\ Carme Torras, and Josep M. Font-Llagunes
}

\begin{abstract}
Wearable sensing technology is proving useful for promoting health and fitness for the general public and athletes, yet few devices are tailored to people with movement impairments. For devices targeting home-based rehabilitation, it is crucial to have robust and non-obtrusive sensors capable of measuring activity for long periods of time outside of a laboratory environment. Studies focusing on continuous monitoring of arm activity during daily life over weeks or months only use IMU sensors or accelerometers at the wrist, and do not capture multi-segment kinematics. In this paper we present the development of the ArmTracker, a fully portable, nonobtrusive and wearable IMU-based motion capture system that can measure arm and torso kinematics for long-periods of time during daily life. We also present the results of a preliminary evaluation of the prototype, carried out with an unimpaired subject and a subject with Becker muscular dystrophy (BMD). Both subjects were asked to wear the ArmTracker device during daily life for 7 hours. We carried out an exploratory graphical analysis with the measured data using three types of movement quality metrics: 1) Range of Motion, 2) Functional Workspace Distribution and, 3) Accelerometry. Results provided an insightful view on the motor function capabilities and limitations of the two subjects. Arm activity of the subject with BMD showed low variability in terms of joint angles and hand positions over the workspace with a clear preference of using his hands in front and below shoulder height. Arm movements of the BMD subject were also slower compared with the unimpaired subject and with a slight preference for using the dominant arm. We plan to extend this study with a larger sample of subjects, and with measurements over several days and weeks to capture representative data on arm activity.
\end{abstract}

\section{INTRODUCTION}

Neuromuscular impairments due to stroke, muscular dystrophy, Parkinson or spinal cord injury, generally result in serious long-term disabilities that hinder the performance of basic activities of daily living (ADL). These disabilities often perpetuate further with secondary physical deterioration caused by the sedentary life style, induced by the disorder, that sets up a vicious cycle of disuse [1], [2], [3]. As a result, quality of life is not only reduced for these patients, but also for their family members and caregivers.

$\dagger$ indicates authors with equal contribution. This work has been partially supported by the Juan de la Cierva - Formación Postdoctoral Fellowship of the Ministerio de Ciencia e Innovación (Ref: FJCI-2017-31754), and by the European Research Council (ERC) under the Horizon 2020 Advanced Grant agreement no. 741930 (project CLOTHILDE).

V.A. Carmona-Ortiz and J.M. Font-Llagunes are with the Department of Mechanical Engineering and Research Centre for Biomedical Engineering, UPC, Barcelona, Spain (victor.antonio.carmona, josep.m.font) dupc.edu

J. Lobo-Prat, J. Van Ruysevelt and C. Torras are with the Institut de Robòtica i Informàtica Industrial, CSIC-UPC, Barcelona, Spain (jlobo, torras) diri.upc.edu

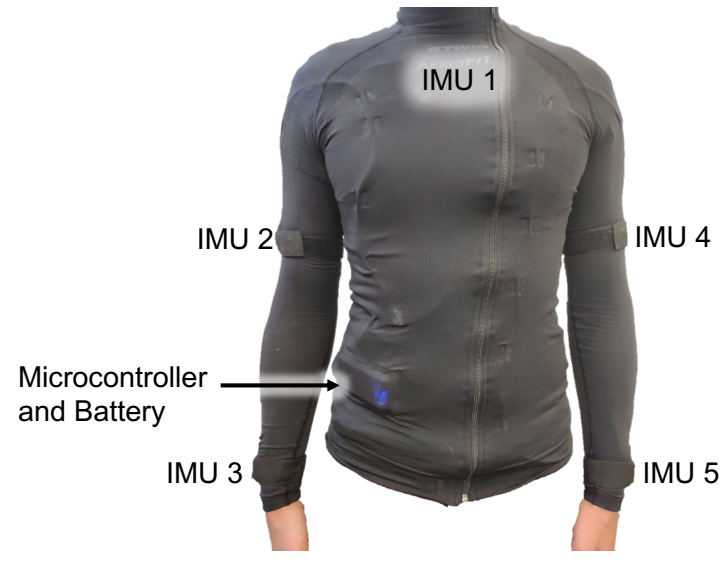

Fig. 1: Prototype of the ArmTracker. This wearable system consists of five IMUs that are integrated on the torso, upper arms and forearms of a lycra shirt. The sensors are wired to a microcontroller with and SD card, and powered by a portable power bank.

The use of wearable sensors such as Inertial Measurement Units (IMU, i.e. combination of accelerometers, gyroscopes and magnetometers) is increasingly popular to analyse movement and track physical activity due to their size, cost, and ease of use [4]. Wearable sensing technology is proving useful for promoting health and fitness for the general public and athletes: recent systematic reviews indicate that even a simple daily pedometer feedback can be effective to increase walking activity and thereby improve body mass index and blood pressure [5]. A plethora of wrist-worn sensors and phone apps for activity monitoring is nowadays marketed and has become a common gear for people exercising for physical fitness. Unfortunately, almost none of these products are tailored to people with movement impairments, and thus they have limited usefulness for them.

While wearable sensors still have a low clinical impact, they potentially have a wide range of clinically relevant applications, including early disorder detection [6], monitoring of disease progression, assessment of treatment efficacy, and safety monitoring (e.g. fall detection) [7]. Focusing on homebased rehabilitation applications, it is crucial to have robust and non-obtrusive sensors capable of measuring activity for long periods of time outside of a laboratory environment. We found that studies focusing on continuous monitoring of arm activity during daily life over weeks or months only use IMU sensors or accelerometers at the wrist, and do not capture multi-segment arm kinematics [8], [9].

The ArmTracker project aims at developing and evaluating a low-cost, comfortable, user-friendly, wearable sensing 


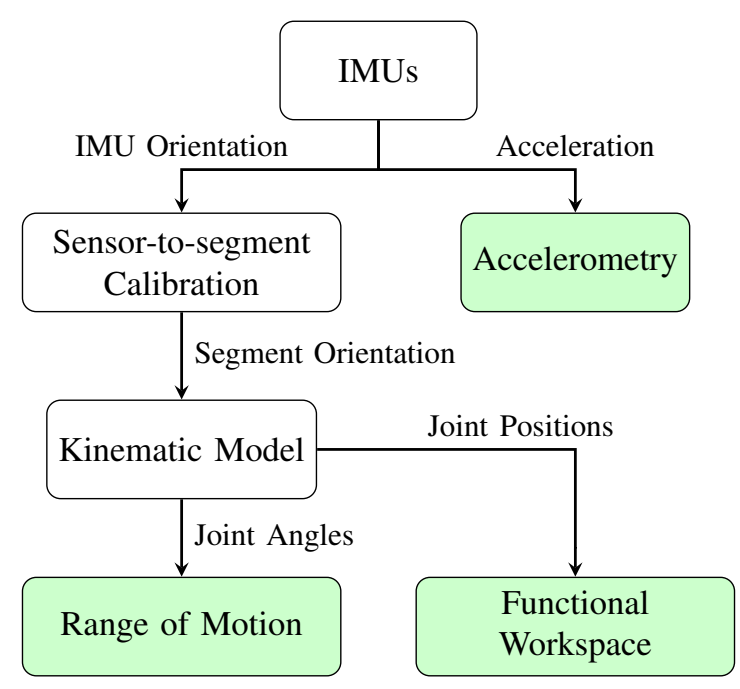

Fig. 2: Flow chart of the data processing and analysis methods used with the ArmTracker system.

system that can gather continuous information on arm kinematics for long periods of time. This information will be used to optimize the amount of arm use during daily life, facilitate subject-specific rehabilitation programs, and ultimately overcome secondary physical deterioration. While the Xsens MVN [10] is a high performance IMU-based motion capture system that is commercially available; we considered that it was not suitable for this particular application. The main reasons being that it is not a fully portable system since it requires a wireless data receiving station, and has a rather high cost.

In this paper we present the development of the first prototype of the ArmTracker (Fig. 1), a non-obtrusive, fully portable, and wearable IMU-based motion capture system that can measure arm and torso kinematics for long-periods of time during daily life. We also present the results of a preliminary evaluation of the prototype carried out with one unimpaired subject and one subject with Becker muscular dystrophy (BMD). Note that the prototype evaluation presented in this paper is a first exploratory graphical analysis towards an in-depth longitudinal study.

\section{Materials And Methods}

\section{A. ArmTracker Prototype}

The prototype of the ArmTracker system is composed of five IMU sensors (BNO055, Bosch Sensortec GmbH), a microcontroller (Teensy 3.6, PJRC.COM, LLC.) with a highspeed SD card (SanDisk Extreme Pro, Western Digital Corporation), and a portable power-bank battery. All the aforementioned components are wired and integrated in a lycra shirt with a front zipper. The IMU sensors are encapsulated in a $3 \mathrm{D}$ printed plastic case and attached with velcro to the inner layer of the lycra shirt at the forearms, upper arms and in the back of the torso (Fig. 1). The lycra shirt also has elastic straps to prevent the IMU sensors from moving. The system is able to measure and store absolute orientation data (in the form of quaternions) from all sensors, as well as triaxial acceleration data from the IMUs on the torso and forearms, at a frequency of $50 \mathrm{~Hz}$ for a maximum period of approximately 8 hours. The cost of the system is approximately $300 €$ and has a total weight of approximately $200 \mathrm{~g}$, which makes the ArmTracker a low-cost, and lightweight system. The data obtained with the ArmTracker are processed (i.e. sensor-tosegment calibration) and used as input in a subject-specific kinematic model to extract joint angles and joint positions (Fig. 2). All the data processing was carried out with a selfdeveloped Matlab script (see Appendix). Measurements of joint angles were used to analyze joint ranges of motion, and the joint positions were used to analyze the functional workspace of the user. In addition, the acceleration data was used to compute actimetry metrics.

\section{B. Sensor-to-Segment Calibration}

We assume that an IMU sensor $S_{i}$ is placed on each body segment $B_{S_{i}}$. In order to obtain accurate orientation measurements of the human segments it is crucial to compensate the misalignment between the coordinate system of the IMU sensors and the coordinate system of the human body segments as defined in the kinematic model. The sensor-tosegment calibration procedure starts by measuring the IMU orientation expressed in the world frame $\left(q_{S_{i}}^{w}\right)$ at time $\tau$, when the subject is in a static and known pose such as the N-pose (i.e. arms straight down) or T-pose (i.e. arms straight to the side). Then, we find the rigid rotation $q_{S_{i}}^{B_{S_{i}}}(\tau)$ between the known body segment orientation $q_{B_{S_{i}}}^{w}(\tau)$ and the measured orientation of the IMU $q_{S_{i}}^{w}(\tau)$ that will allow us to calculate the orientation of the body segments $q_{B_{S_{i}}}^{w}$ from the IMU measurements $q_{S_{i}}^{w}$ :

$$
\begin{aligned}
q_{B_{S_{i}}}^{S_{i}}(\tau) & =q_{S_{i}}^{w *}(\tau) \otimes q_{B_{s_{i}}}^{w}(\tau) \\
q_{B_{S_{i}}}^{w} & =q_{S_{i}}^{w} \otimes q_{B_{S_{i}}}^{S_{i}}(\tau),
\end{aligned}
$$

where $*$ indicates the complex conjugate of a quaternion, and $\otimes$ indicates the quaternion multiplication operator. Note that if the relative orientation between the IMU and the body segment changes, this procedure needs to be repeated.

\section{Kinematic Model}

The orientations of the body segments $q_{B_{S_{i}}}^{w}$ were used as input for a kinematic model to calculate joint angles and joint positions of the elbow and wrist joints, using the subjectspecific length of the upper arm and forearm. The joints were modelled as spherical joints with 3 degrees of freedom (DOF). To build the kinematic model we first calculated the relative orientations between adjacent body segments $\left(B_{S_{1}}\right.$, $B_{S_{2}}$ ) using:

$$
q_{B_{S_{2}}}^{B_{S_{1}}}=q_{B_{S_{1}}}^{w *} \otimes q_{B_{S_{2}}}^{w}
$$

Second, the quaternions expressing the relative rotation between body segments were converted to rotation matrices $R\left(q_{B_{S_{2}}}^{B_{S_{1}}}\right)$. Third, the resulting rotation matrices together with the translation vectors that describe the vector from joint 1 to joint $2\left(\mathbf{p}_{J_{2}}^{J_{1}}\right)$ were used to build an homogeneous transformation matrix:

$$
H_{B_{S_{2}}}^{B_{S_{1}}}=\left[\begin{array}{cc}
R\left(q_{B_{S 2}}^{B_{S_{1}}}\right) & \mathbf{p}_{J_{2}}^{J_{1}} \\
0 & 1
\end{array}\right]
$$




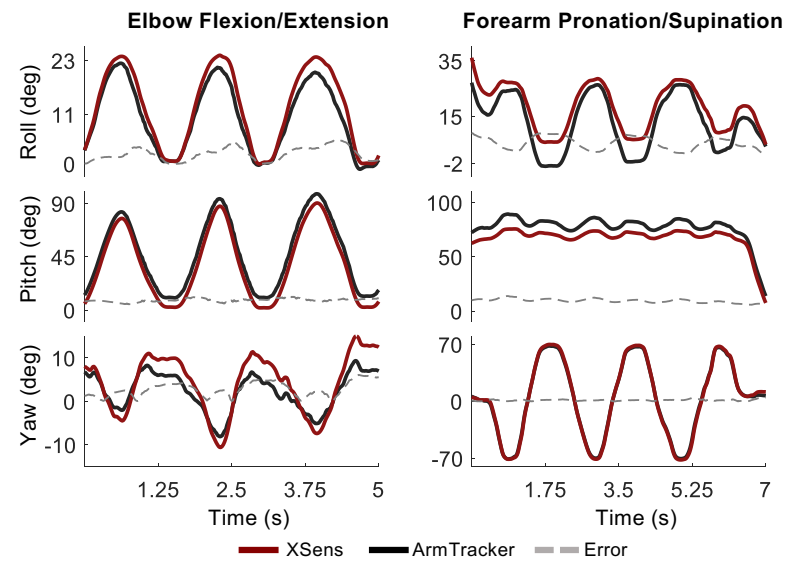

Fig. 3: Results of comparing the Euler angles (roll, pitch, yaw) obtained with the ArmTracker (black) and the Xsens (red) systems during elbow flexion/extension and forearm pronation/supination. The dashed gray line indicates the error between the two systems.

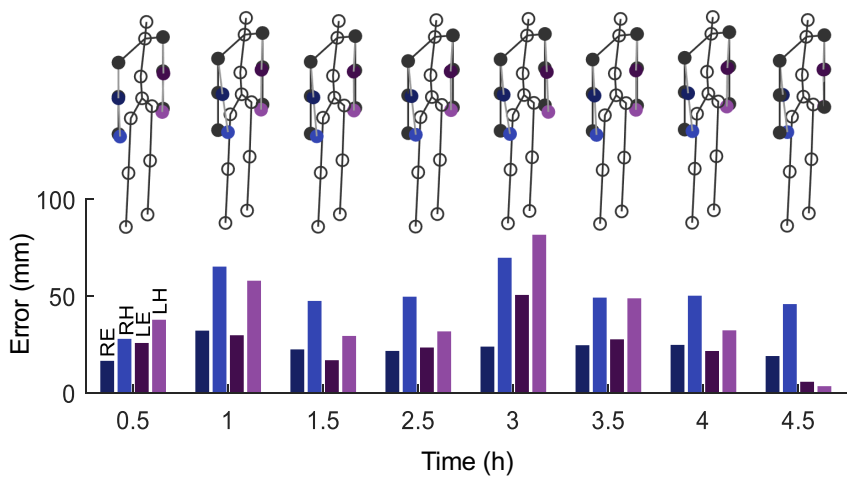

Fig. 4: Results of comparing several N-Poses over time with respect to the initial N-Pose. The bar plot shows the position deviation for the right elbow (RE), right hand (RH), left elbow (LE) and left hand $(\mathrm{LH})$.

The concatenation of the resulting homogeneous transformation matrices was used to obtain the positions of the wrist and elbow joints relative to the torso segment.

\section{System Validation}

To validate the accuracy of the ArmTracker, we compared our prototype to a commercially available IMU-based motion capture system (i.e. Xsens MVN [10]). We carried out several measurements of shoulder and elbow single joint movements wearing both systems at the same time to measure the deviation in position and orientation between the data obtained from both systems. In order to compare the orientation difference we calculated the joint Euler angles.

In addition, we also tested the system stability when measuring and storing data. In the first test, the ArmTracker system was let still for 12.5 hours and we calculated the drift of sensors by comparing samples from the beginning and end of the capture time. In the second test, the unimpaired subject was asked to wear the ArmTracker system for 4.5 hours during his daily life and periodically repeat the static $\mathrm{N}$-pose. These data were used to calculate the deviations in joint positions for each of the $\mathrm{N}$-poses in order to assess the reliability of the data during captures.

\section{E. Movement Quality Metrics}

Three types of metrics were computed to analyze the quality of the upper limb movements: 1) Range of Motion, 2) Functional Workspace Distribution, and 3) Accelerometry. These metrics where calculated with data recorded from an unimpaired subject (male, 24 years old, dominant arm: right), and a subject with BMD (male, 25 years old, Brooke scale 2, dominant arm: left). Both subjects were asked to wear the ArmTracker device during daily life for 7 hours (i.e. approximately from 10 a.m. to 5 p.m.).

1) Range of motion: Shoulder elevation and elbow flexion/extension angles were obtained calculating the angles between the longitudinal vectors of the upper arm (u) and torso (v), and between the upper arm (u) and the forearm (v), respectively. We used the cosine rule to extract the angles of interest as in [11]:

$$
\alpha=\arccos \left(\frac{\mathbf{u} \cdot \mathbf{v}}{\|\mathbf{u}\|\|\mathbf{v}\|}\right) .
$$

2) Functional Workspace Distribution: The position of the hand over time was used to assess the functional workspace distribution. Isolated areas of hand activity were filtered by calculating the centroid of all hand positions, and removing the $10 \%$ of the points that were further out from the centroid. The workspace was divided in 8 different regions using the shoulder height and the shoulder-elbow distance as reference points. We quantified, the percentage of time that the hand of the user remained inside each specific region.

3) Accelerometry: Finally, the recorded wrist acceleration data were used to extract accelerometry metrics. We calculated unilateral activity, bilateral magnitude, and magnitude ratio following the methodology proposed in [12], [13]. For each data window of one second (i.e. every 50 samples) we computed the average acceleration for each arm. Afterwards, these values were transformed into counts, defining 1 count as $0.0163 \mathrm{~m} / \mathrm{s}^{2}$. From the count values, unilateral activity was extracted inspecting the count values for each arm. If the activity count of one window for one of the two arms was 0 , that window was classified as unilateral activity. The bilateral magnitude was extracted as the sum of the counts for each window for both arms. Lastly, from the unilateral arm activity measures, we computed the magnitude ratio dividing the unilateral activity values of the dominant arm of the participants by the activity counts of the contralateral arm, and calculated the natural logarithm of that value. The magnitude ratio expresses the dominance of each arm. Negative values of the magnitude ratio imply higher activity for the dominant arm of the subject, while positive values of the magnitude ratio indicate higher activity for the non dominant arm, and 0 implies that both arms had the same contribution.

\section{RESULTS}

\section{A. System Validation}

The joint positions obtained with the ArmTracker and the Xsens MVN systems showed maximum position deviations of $43.5 \mathrm{~mm}$ and $41.8 \mathrm{~mm}$ for the right elbow and wrist joints, 

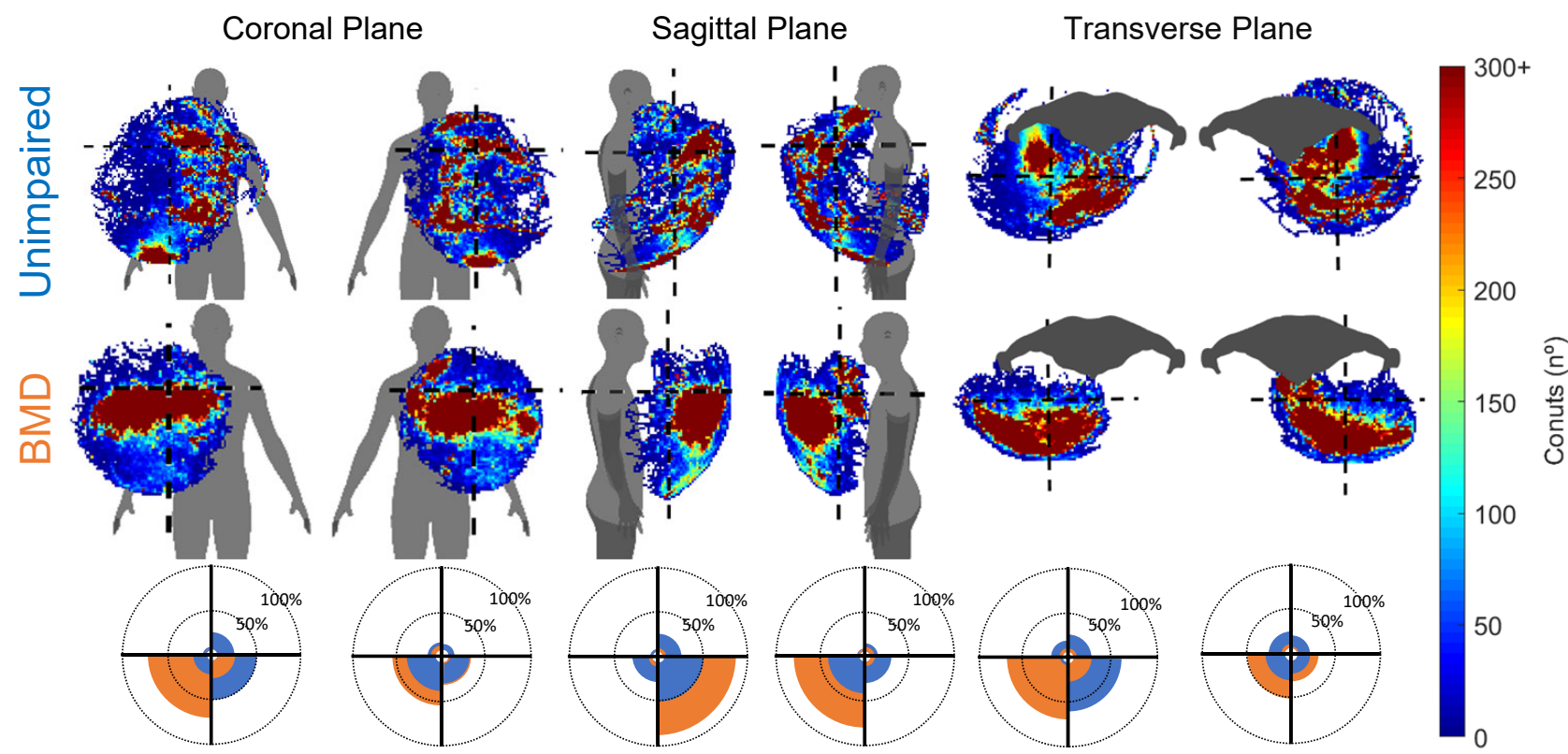

Fig. 5: Density plots for each anatomical plane showing the functional workspace distribution of the right and left wrist joint for the subject with BMD (middle) and the unimpaired subject (top). At the bottom, the circular bar plots show the percentage of time that the wrist joint was in each region.

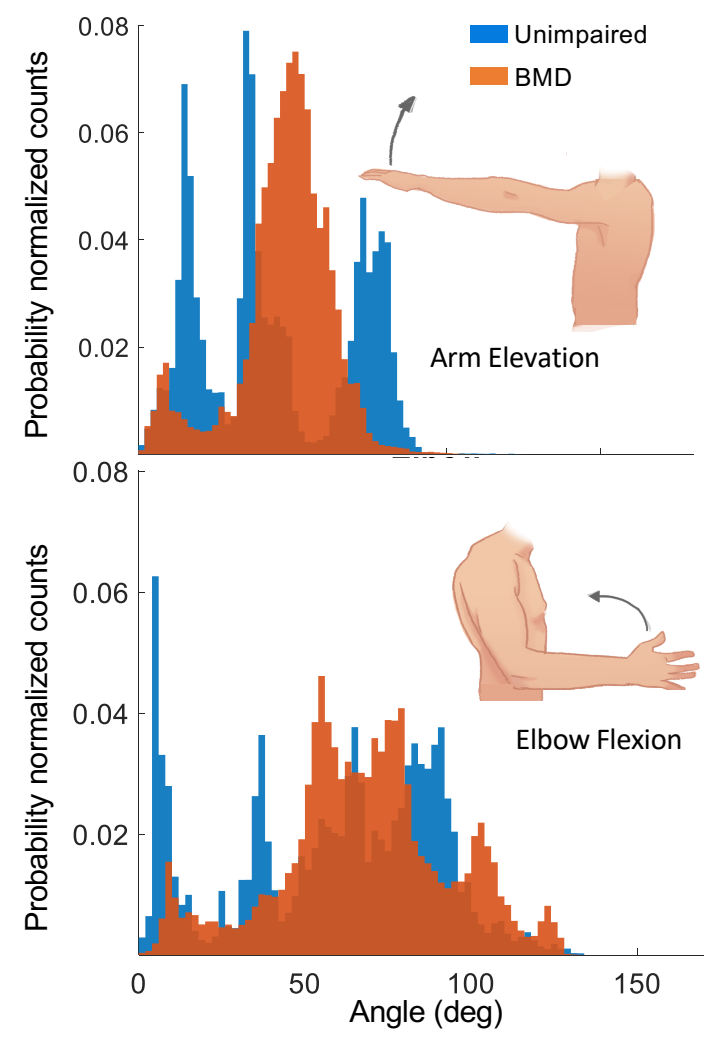

Fig. 6: Histograms of arm elevation (top) and elbow flexion/extension (botton) angles for the dominant arms of the subject with BMD (orange) and the unimpaired subject (blue).

respectively. The other joint positions presented errors in the range of 12.88 to $25.99 \mathrm{~mm}$ for the left elbow, and 8.15 to $34.9 \mathrm{~mm}$ for the left wrist. Regarding the comparison of joint angles, maximum RMSE values of 5.59, 9.97 and 5.17 $\mathrm{deg}$. were found for shoulder abduction, shoulder flexion and

shoulder internal/external rotations, respectively. As for the forearm orientation, we found maximum RMSE values of 9.68 and $8.44 \mathrm{deg}$. or elbow flexion/extension and forearm pronation/supination movements (Fig. 3). Static stability tests performed during a $12 \mathrm{~h}$ measurement showed a maximum mean error of 0.0091 of the quaternion values (which can range from -1 to 1 ), and a $0.3 \%$ of data loss. Dynamic stability tests performed during a $4 h$ measurement showed a maximum joint position error of $81.7 \mathrm{~mm}$ between $\mathrm{N}$-poses for the left hand position estimates (Fig. 4).

\section{B. Exploratory Graphical Analysis}

Next we show the results of the exploratory graphical analysis carried out on the three types of movement quality metrics with the $7 h$ of data recorded from the unimpaired and the BMD subjects. The purpose of this analysis was to visually identify arm movement differences between the two subjects and not the implementation of statistical models.

1) Range of Motion: We found that arm elevation movements of the subject with BMD presented a narrow distribution centered at $50 \mathrm{deg}$, whereas the healthy subject showed a more spread distribution, presenting three peaks at 25, 40 and $70 \mathrm{deg}$. (Fig. 6). The arm elevation range of motion was larger for the unimpaired subject. Elbow flexion/extension angles of the subject with BMD presented a distribution centered at $60 \mathrm{deg}$. with smaller peaks at 10 and $100 \mathrm{deg}$. On the other hand, the healthy subject presented high peaks at 10, 40, 60 and $130 \mathrm{deg}$. The elbow flexion/extension range of motion was similar for both subjects.

2) Functional Workspace Distribution: The subject with BMD presented a smaller workspace volume compared to the unimpaired participant (BMD: $0.09 \mathrm{~m}^{3}$, unimpaired: 0.2 


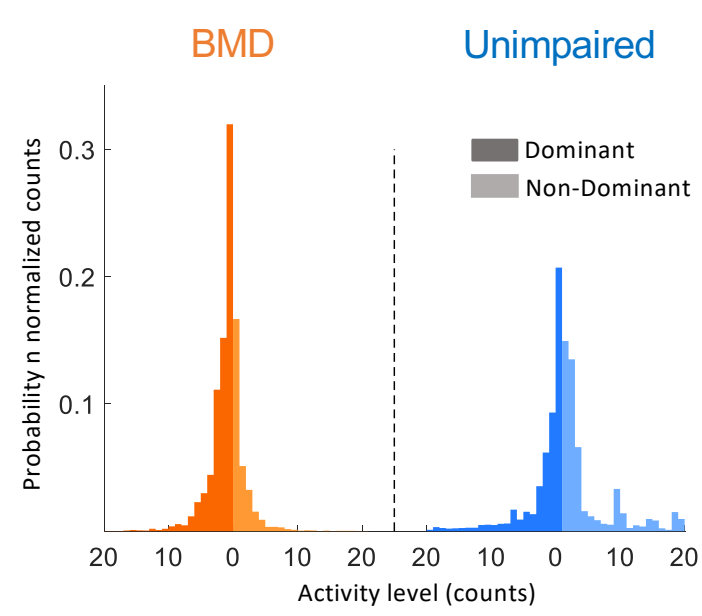

Fig. 7: Histogram of the unilateral arm activity for the dominant (dark color) and non-dominant (faded color) arms of the subject with BMD (orange) and the unimpaired subject (blue). Activity level is measured in counts $\left(1\right.$ count $\left.=0.0163 \mathrm{~m} / \mathrm{s}^{2}\right)$. This representation method was proposed by [12].

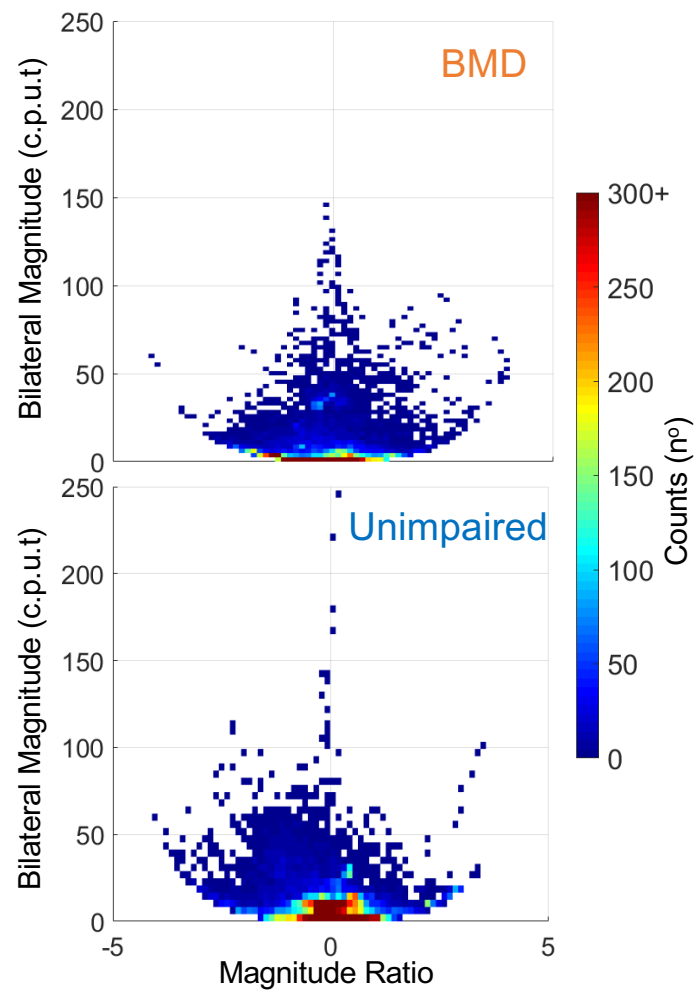

Fig. 8: Bilateral Magnitude (expressed in counts per unit time) as function of Magnitude Ratio for the subject with BMD (top) and the unimpaired participant (bottom). Bilateral magnitude expresses the activity level of both arms and magnitude ratio indicates if the activity is done by the non-dominant (positive values) or dominant (negative values) arm. This representation method was proposed by [13].

$\left.m^{3}\right)$. Specifically, we can see from the data shown in the transverse plane that the unimpaired subject explored both proximal and distal regions (even reaching posterior areas), while the subject with BMD only explored distal regions. The activity of the subject with BMD was concentrated slightly below the shoulder height for $90 \%$ of the time (see sagittal plane in Fig. 5) and spread between the lateral (70\%) and medial $(25 \%)$ regions (see coronal and transverse planes in Fig. 5). The unimpaired subject presented a more diverse workspace with three main visited areas that were vertically scattered at three heights: over the shoulder, mid torso, and waist height (see sagittal plane in Fig. 5). Note that the fact that the workspace is more diverse brings down the percentages of time spent in each region. The most visited regions are below the shoulder medial (50\%), followed by over the shoulder medial (26\%) regions. A noticeable differences between subjects was their use of medial and lateral regions: while the subject with BMD spent $70 \%$ of the time on the lateral regions, the unimpaired participant spent most of the time on the medial regions.

3) Accelerometry: Unilateral arm activity of the subject with BMD was considerably larger for the dominant arm (71.1\%) compared to the non-dominant arm $(28.9 \%$; Fig. $7)$. We also found that the majority of the counts were concentrated at low activity levels (i.e. below 10). In contrast, the unilateral arm activity of the unimpaired subject was equally distributed for both dominant $(50.1 \%)$ and nondominant arms (49.9\%). In addition, the activity level of the unimpaired subject reached higher levels (i.e. up to 20), especially for the dominant arm, compared to the subject with BMD. Regarding the bilateral activity metric (Fig. 8), the healthy subject presented a higher bilateral magnitude, indicating faster arm motions compared to the subject with BMD. In terms of arm dominance, the unimpaired subject showed that movements with high acceleration (high bilateral magnitude) were done with both arms (magnitude ratio $=0$ ) or by the dominant arm (magnitude ratio $<0$ ). For that same subject, low acceleration movements (low bilateral magnitude) did not show a clear dominance (magnitude ratio $=0$ ). In contrast, the fast arm movements of the subject with BMD did not present a clear dominance: activity was concentrated at magnitude ratios close to 0 . Yet, slow movements had a clear tendency for the dominant arm (magnitude ratio $<0$ ).

\section{DISCUSSION}

Results of the system validation showed that the ArmTracker system is capable of providing long-term, stable data, so that consistent measurements can be obtained for long periods of time. As for the robustness of the output, the joint position deviations during the uncontrolled 4.5 hours of measurement were found to be below $80 \mathrm{~mm}$. However, note that besides system inaccuracies caused by magnetic interference [14] or relative movement between the IMU sensors and the body segments [15], probably there was also human error when repeating the N-poses. Errors due to relative motion between the IMUs and the corresponding segments can be reduced by repeating the calibration procedure periodically. The results of the comparison test between the ArmTracker and the Xsens MVN showed acceptable deviations in position and orientation, specially taking into account that the purpose of the system is to monitor arm activity and not to perform high precision motion capture. 
We were able to use the ArmTracker system to monitor the arm activity during daily life for a long period of time (i.e. 7 $h$ ) with a subject with BMD and an unimpaired subject. At the end of the measurements both subjects mentioned that the system was comfortable and easy to use.

The movement quality metrics used for the exploratory graphical analysis provided an insightful overview on the motor function capabilities and limitations of the two subjects. The subject with BMD showed low variability in terms of joint angles (Fig. 6) and hand positions over the workspace, with a clear preference of having his hands in front and below the shoulder height (Fig. 5). This is probably related to the fact that both subjects carried out office work for most part of the monitoring period. Comparing the activity of both arms, we found very similar workspace areas for the left and right sides (see Fig. 5), with a slight preference for using more the dominant arm, specially during slow movements (see Figs. 7 and 8). In general, the unimpaired subject showed more variability in all metrics compared to the subject with BMD. We found that the unimpaired subject had three main postures that were noticeable when analyzing the arm elevation and elbow flexion/extension angles, as well as the functional workspace distribution plots. In terms of accelerometry metrics we found, as expected, that the unimpaired subject performed movements at higher accelerations compared to the subject with BMD with a clear preference for the dominant arm. It is worth noting that, since the measurements carried out in this study were only performed during one single day, the present results are not sufficient to fully characterize the participants' behaviour.

Future work will include carrying out a longitudinal study of several days and weeks to obtain representative results on arm movement quality, as well as increasing the number of study participants to be able to implement statistical models on the data obtained. Future work will also involve exploring the possibility of integrating muscular activity measurements (i.e. electromyography) to be able to estimate muscle fatigue. Finally, we also plan to use the ArmTracker system in combination with assistive technology, such as mobile arm supports, to analyze their effectiveness and usability.

\section{Conclusions}

In this paper we presented the development of the ArmTracker, a non-obtrusive and wearable IMU-based motion capture system that can measure multi-segment kinematics. Results of the system validation showed that the ArmTracker system is capable of providing stable and accurate data on upper body kinematics, so that consistent measurements on arm activity outside of laboratory environments can be obtained for long periods of time. The ArmTracker was successfully used to measure the arm activity during daily life for $7 h$ with one subject with BMD and one unimpaired subject. The exploratory graphical analysis on range of motion, functional workspace distribution, and accelerometry metrics provided an insightful view on the motor function capabilities and limitations of the two subjects.

\section{APPENDIX}

A detailed explanation and sample code of the kinematic model are available in: https://github.com/ jloboprat/IMU_Kinematic_Model

\section{ACKNOWLEDGEMENTS}

We would like to thank the support of Joan Solà [16] during the development of the kinematic model.

\section{REFERENCES}

[1] M. Jansen, N. van Alfen, A. C. H. Geurts, and I. J. M. de Groot, "Assisted bicycle training delays functional deterioration in boys with Duchenne muscular dystrophy: the randomized controlled trial "no use is disuse"," Neurorehabilitation and Neural Repair, vol. 27, pp. 816827, Dec. 2013.

[2] S. A. Billinger, R. Arena, J. Bernhardt, J. J. Eng, B. A. Franklin, C. M. Johnson, M. MacKay-Lyons, R. F. Macko, G. E. Mead, E. J. Roth, et al., "Physical activity and exercise recommendations for stroke survivors: a statement for healthcare professionals from the american heart association/american stroke association," Stroke, vol. 45 , no. 8 , pp. 2532-2553, 2014.

[3] K. A. M. Ginis, A. L. Hicks, A. E. Latimer, D. E. R. Warburton, C. Bourne, D. S. Ditor, D. L. Goodwin, K. C. Hayes, N. McCartney, A. McIlraith, P. Pomerleau, K. Smith, J. A. Stone, and D. L. Wolfe, "The development of evidence-informed physical activity guidelines for adults with spinal cord injury," Spinal Cord, vol. 49, pp. 10881096, Nov. 2011.

[4] P. Bonato, "Wearable sensors/systems and their impact on biomedical engineering," IEEE Engineering in Medicine and Biology Magazine, vol. 22, no. 3, pp. 18-20, 2003.

[5] D. M. Bravata, C. Smith-Spangler, V. Sundaram, A. L. Gienger, N. Lin, R. Lewis, C. D. Stave, I. Olkin, and J. R. Sirard, "Using pedometers to increase physical activity and improve health: a systematic review," JAMA, vol. 298, pp. 2296-2304, Nov. 2007.

[6] J. Barth, J. Klucken, P. Kugler, T. Kammerer, R. Steidl, J. Winkler, J. Hornegger, and B. Eskofier, "Biometric and mobile gait analysis for early diagnosis and therapy monitoring in parkinson's disease," in 2011 Annual International Conference of the IEEE Engineering in Medicine and Biology Society, pp. 868-871, IEEE, 2011.

[7] S. Khojasteh, J. Villar, C. Chira, V. González, and E. De La Cal, "Improving fall detection using an on-wrist wearable accelerometer," Sensors, vol. 18, no. 5, p. 1350, 2018.

[8] Q. Wang, P. Markopoulos, B. Yu, W. Chen, and A. Timmermans, "Interactive wearable systems for upper body rehabilitation: a systematic review," Journal of Neuroengineering and Rehabilitation, vol. 14, no. 1, p. 20, 2017.

[9] P. Maceira-Elvira, T. Popa, A.-C. Schmid, and F. C. Hummel, "Wearable technology in stroke rehabilitation: towards improved diagnosis and treatment of upper-limb motor impairment," Journal of Neuroengineering and Rehabilitation, vol. 16, no. 1, p. 142, 2019.

[10] D. Roetenberg, H. Luinge, and P. Slycke, "Xsens mvn: Full 6dof human motion tracking using miniature inertial sensors," Xsens Motion Technologies BV, Tech. Rep, vol. 1, 2009.

[11] I. S. Howard, J. N. Ingram, K. P. Kording, and D. M. Wolpert, "Statistics of natural movements are reflected in motor errors," Journal of Neurophysiology, vol. 102, no. 3, pp. 1902-1910, 2009.

[12] R. R. Bailey, J. W. Klaesner, and C. E. Lang, "Quantifying realworld upper-limb activity in nondisabled adults and adults with chronic stroke," Neurorehabilitation and neural repair, vol. 29, no. 10, pp. 969-978, 2015.

[13] R. R. Bailey, J. W. Klaesner, and C. E. Lang, "An accelerometry-based methodology for assessment of real-world bilateral upper extremity activity," PloS one, vol. 9, no. 7, p. e103135, 2014.

[14] X. Robert-Lachaine, H. Mecheri, C. Larue, and A. Plamondon, "Effect of local magnetic field disturbances on inertial measurement units accuracy," Applied Ergonomics, vol. 63, pp. 123-132, 2017.

[15] A. Leardini, L. Chiari, U. Della Croce, and A. Cappozzo, "Human movement analysis using stereophotogrammetry: Part 3. soft tissue artifact assessment and compensation," Gait \& posture, vol. 21, no. 2, pp. 212-225, 2005.

[16] J. Sola, "Quaternion kinematics for the error-state kalman filter," arXiv preprint arXiv:1711.02508, 2017. 